\title{
A Self-Consistent Turbulence Generated Scenario for L-H Transition.
}

\author{
Y.Z. Zhang and S.M. Mahajan \\ Institute for Fusion Studies \\ The University of Texas at Austin \\ Austin, Texas 78712
}

DOE/ET/53088--575

DE93 001853

\begin{abstract}
The turbulence-induced ion banana polarization current associated with steep ion temperature gradients is explored as a possible mechanism for generating poloidal momentum at the tokamak edge. In the light of a recently developed two-dimensional turbulence theory, one can obtain a simple closed expression relating this current (determined by turbulence levels) to the derivatives of the poloidal rotation speed. A self-consistent system, then, emerges, if we balance the turbulence-induced poloidal momentum with that dissipated by viscosity. Under suitable conditions this system may show a bifurcation controlled by a parameter dependent on temperature gradients. Both the bifurcation point, and the shear layer width are predicted for a prescribed flow in terms of a scale characterizing the nonlinearity of viscosity. The crucial relevance of the flow parity with the turbulence scenario is analyzed.
\end{abstract}

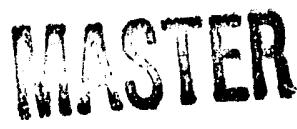


The physics of L-H transitions ${ }^{1}$ is one of the most important and interesting topics of current fusion research. It is one of those topics where theoretical challenges and practical importance are all tied together. The transition itself is characterized by a sudden increase in poloidal rotation near the plasma edge, immediately followed by a rapid suppression of turbulence, resulting in a significant improvement in the local confinement. ${ }^{2-8}$ To understand the nature of $\mathrm{L}-\mathrm{H}$ transitions, one seeks answers to questions in two broad catagories: (1) the turbulence suppression due to an increase in the poloidal shear flow, ${ }^{9-14}$ and (2) mechanism(s) for the generation of the required flow. ${ }^{9,11,12,15-19}$ There have been some efforts to formulate a unified, self-consistent theory, which could explain simultaneously the improved confinement, and the flow generation. At this stage, however, we are far from a satisfactory understanding, in particular of the mechanism(s) for the generation of shear flow. It is the purpose of this Letter to examine a possible physical mechanism, and to discuss the properties of a self-consistent bifurcation system with a built-in suppression of turbulence due to the shear flow, which, in turn, was generated by the turbulence. More specifically, our model is constructed on the basis of the poloidal momentum balance expressed in terms of radial currents. The radial current generated by turbulence is proportional to the turbulence level, with a coefficient associated with and dependent on the piasma equilibrium (the so-called control parameter of bifurcation). Therefore, it is not surprising that even though the turbulence levels are suppressed by the shear flow, the radial current can still be large, because of the large control parameter as well as due to the nonlinearity of the dissipation that yields a counter-radial current to balance the driving one.

We would like to state that in the cold ion limit, a turbulence-based mechanism for the generation of the radial current already exists in the literature; ${ }^{20,17}$ the current is related to the net energy flow in the radial direction. For the weakly dissipative drift waves, for instance, the dominance of the ion sound effect makes the wave energy flow in either direction away 
from the rational surface, i.e., in the radially inward as well $: \cdots$ the radially outward direction. In the region between two rational surfaces, the energy flow contributed by the two modes is in opposite directions. It is clear that due to this "pairing," ${ }^{21}$ a strong reduction in the net radial flow should take place. This reduction is easily measured by the ratio of the microscopic scale length characteristic of the individual modes $\sim \rho_{s}$, the ion Larmor radius at the electron temperature, to the overall macroscopic scale length associated with the variation in the wave amplitude. The phenomenon is analogous to the diamagnetic drift being a fraction $\rho / L$ of the thermal speed, where $\rho$ is the Larmor radius, and $L$ is the equilibrium gradient length.

For the dissipative drift waves $22-24$ (where the ion sound effect is not important), and also for other modes associated with strong dissipation such as the rippling mode ${ }^{25}$ and the resistive interchange mode, ${ }^{26}$ the wave energy is "locked" around the rational surface because these modes are essentially standing waves. Thus, the mechanisms relying or the radial energy flow cease to be effective for generating a radial current in the cold ion limit. In this case the turbulence-induced radial current could originate from the warm ion inertia $\left[\left\langle\tilde{\mathbf{u}}_{D_{\mathbf{1}}} \cdot \nabla \tilde{\mathbf{u}}_{E}\right\rangle\right.$, where $\tilde{\mathbf{u}}_{D_{\mathbf{1}}}$ is the fluctuating ion diamagnetic flow, $\tilde{\mathbf{u}}_{E}$ is the fluctuating $\mathbf{E} \times \mathbf{B}$ flow, and $\langle\ldots\rangle$ is the ensemble average on turbulence]. In slab geometry, the calculation (neglecting ion temperature fluctuation) is very similar to that for the "quasilinear" particle flux, and the calculated current turns out to be

$$
J_{r}^{(\mathrm{pol})}=\frac{e}{4} \cdot \frac{d}{d r} \rho_{i}^{2}\left(\Gamma_{r} \frac{d}{d r} \log \frac{n}{T_{e}}-S\right)
$$

where $\Gamma_{r}$ is the turbulence-induced particle flux, $S$ is the particle source $\left(\equiv d \Gamma_{r} / d r\right), e$ is the electron charge, $\rho_{i}$ is the ion Larmor radius, $n$ is the plasma density, and $T_{e}$ is the electron temperature.

From Eq. (1), and also from earlier cold ion calculations, it can be seen that schematically, 
the turbulence-induced radial current for either case can be written in the form:

$$
J_{r}^{(\mathrm{pol})} \sim e \Gamma_{r}\left(\frac{\rho}{\Delta}\right)^{2}
$$

where $\Delta$ is an appropriate macroscopic scale length (amongst those describing the radial variation of equilibrium density, temperatures and wave amplitude), and $\rho$ can either be $\rho_{i}$ or $\rho_{s}$, depending upon which is more relevant to the physical process involved. Because $\rho / \Delta \ll 1$, this radial current, however, is found to be too small to balance the viscous dissipation for generating experimentally observed poloidal rotation corresponding to the parameters of L-H transition on tokamaks.

The turbulence-induced ion polarization current can be greatly enhanced by finite ion banana width, if the turbulence correlation length along the field line is longer than the banana length. In this work, we assume that the aforementioned condition is satisfied. As a result, the Larmor radius $\rho$ in Eq. (2) should be replaced by the "poloidal" Larmor radius $\rho_{\mathrm{i} \theta},{ }^{27}$ as the edge ions enter into the banana regime. The enhanced current is thus written as

$$
J_{r}^{(\mathrm{pol})} \sim e \epsilon^{*} \Gamma_{r}\left(\frac{\rho_{\mathrm{i} \theta}}{\Delta}\right)^{2}
$$

where $\epsilon^{*}$ is composed of the three small factors: (a) $\epsilon_{1}$, the ratio of trapped particle density to the total density, which is subject to be greatly reduced from its normal value $\sqrt{\epsilon}(\epsilon$ is the inverse aspect ratio) by a large $\mathbf{E} \times \mathbf{B}$ flow, the so-called electrostatic detrapping, ${ }^{28}$ even if the edge ions still remain in the banana regime, (b) $\epsilon_{2}$, the bootstrap diamagnetic effects associated with the warm ions, which is essentially $\sqrt{\epsilon}$, and (c) $\epsilon_{3}$, the ratio of wave radial correlation length to the banana width, if the former were to be smaller than the latter, otherwise $\epsilon_{3} \leq 1$. The radial current given by Eq. (3) is proportional to the turbulence intensity via $\Gamma_{r}$. The appearance of a shear flow will modify the intensity by ${ }^{13,14}$

$$
\left\langle|\varphi|^{2}\right\rangle=\frac{\left\langle|\varphi|^{2}\right\rangle_{0}}{1+\left|t_{c 0} \frac{d v}{d r}\right|^{\mu}}
$$


where $\mu$ is a number between 1 to $2, t_{c 0}$ is the decorrelation time of the turbulence, $\left\langle|\varphi|^{2}\right\rangle_{0}$ is the turbulence intensity (the subscript 0 stands for the quantity without shear flow). The turbulence-induced radial current drives a poloidal momentum with flow $v$, which in turn induces a counter-radial current through the agency of the viscosity $\Theta$. The requirement for a net zero radial current then yields a differential equation for the poloidal velocity:

$$
\frac{\alpha}{1+\left|\frac{d v}{d x}\right|^{\mu}}=\Theta[v]
$$

where $\Theta[v]$ is a nonlinear function, approaching linear as $v$ becomes small, and is characterized by a local maximum of $\Theta[v]$ at $v=v^{*}$. In Eq. (5), $x$ is the radial coordinate normalized to $r_{s} \equiv v^{*} t_{c 0}$, and $\alpha$ is a quantity determined by the characteristics of the turbulence-induced drive and the dissipation due to viscosity. An example for $\Theta[v]$ is provided by the kinetic model of Shaing et al., ${ }^{11}$ for which $v^{*} \sim a \nu$, and $a$ and $\nu$ are respectively the minor radius, and the ion collision frequency. Making use of Eq. (3) and the neoclassical viscosity in the small $v$ limit, we obtain $\alpha=\bar{\epsilon}^{*} \Gamma_{r, 0} \rho_{i, \theta} R / \epsilon n v^{*} \Delta^{2}$, where $\Gamma_{r, 0}$ is the radial particle flux without shear flow (the local Low-mode radial $f: \because x), \bar{\epsilon}^{*}$ is the $v$-independent part of $\epsilon^{*}$, and $R$ is the major radius.

For this first-order differential equation (even in $x$ ), the choice of the boundary condition at $x=0$ is imposed by the parity of the flow. In this Letter we consider only the even parity flows like those observed in DIII-D experiments, ${ }^{29,30}$ i.e., the double shear layer structures with essentially symmetric flow around the peak. We choose the origin $(x=0)$ to be precisely at the peak, i.e., $|d v / d x|_{x=0}=0$. This choice implies

$$
\Theta[v(0)]=\alpha
$$

where $v(0)$ is the peak of flow in the shear layer. In order that a solution of Eq. (6) may exist for large $\alpha, \Theta[v]$ must have a second rising-stage ( $C-D$ sector of Fig. 1), which may arise either from the possible electrostatic detrapping, or from a real increase of viscosity at 
large flow. In this Letter, without offering any derivation, we assume tha: $\Theta[v]$ does indeed have the qualitative form depicted in Fig. 1.

It can be readily shown that for those $v(0)$ s corresponding to the positive $d \Theta / d v$ sector [ $A-B, C-D$, the bold solid curve sector in Fig. 1], the solutions of Eq. (5) must have a convex configuration, i.e., $v(x) \leq v(0)$. In contrast, for those $v(0)$ s within the negative $d \Theta / d v$ sector $[B-C$ the thin solid curve sector in Fig. 1], the solutions show a concave configuration, i.e., $v(x) \geq v(0)$. The qualitative features of these two configurations for the same $\alpha\left(<\Theta\left[v^{*}\right]\right.$, labeled by $v(0)=v_{-}$and $v_{+}$respectively) are illustrated in Fig. 2, where the emphasis is on the distinction in topology. Both the local maximum and minimum $[B$ and $C$ ] are singular points. At these points, there are no acceptable solutions which may satisfy the boundary condition, $|d v / d x|_{x=0}=0$. In a sense, the discontinuity in topology (from convex to concave and vice versa) forbids the system evolving continuously from one to the other. As a result, the transition must take place, just as these singularities are reached. For increasing $\alpha$, the transition occurs at $B[B \rightarrow D]$, while for falling $\alpha$, it must happen at $C[C \rightarrow A]$, implying "hysterisis" in the system. For this reason, the concave configuration of $v(x)$ would not pertain in the normal machine operation.

The above discussion clearly yields the $\mathrm{L} \rightarrow \mathrm{H}$ bifurcation condition at $\alpha \sim 1$. The solution of Eq. (5) also yields a radial structure of shear flow (notice that the solution for the radial structure is meaningful only for $x \leq 1$ ). Regardless of the details of the radial structure, Eq. (5) implies that the width of physical shear layer is essentially measured by $r_{s}$.

The system, in fact, also suggests a $v^{*}$-model independent relation between the measured quantities at the $\mathrm{L} \rightarrow \mathrm{H}$ transition. By substituing $v^{*}=r_{s} / t_{\mathrm{co}}$ into $\alpha \sim 1$, we readily obtain the condition for $\mathrm{L} \rightarrow \mathrm{H}$ transition:

$$
\frac{\Delta r_{s}}{R \rho_{i \theta}} \leq \frac{\bar{\epsilon}^{*} t_{c 0} \Gamma_{r, 0}}{\bar{\epsilon} n \Delta} \sim \frac{\bar{\epsilon}^{*}}{\epsilon \bar{k}_{\perp}^{2} \Delta^{2}}
$$

where $\bar{k}_{\perp}$ is the averaged perpendicular wave number of the turbulence spectrum. It is 
noticeable that in order for $\alpha$ to be sufficiently large, the edge ions should be either in, or near the border of the banana regime. Further information can also be drawn, if a specified nonlinear viscosity model is provided. For example, assuming a $v^{*} \sim a \nu,{ }^{11}$ the shear layer width becomes $r_{s} \sim a t_{c 0} \nu$, and Eq. (7) reduces to $\Delta^{2} \nu / \rho_{i \theta} \leq \bar{\epsilon}^{*} \Gamma_{r, 0} / \epsilon^{2} n$.

Finally, we would like to point out that the radial flow structure with odd parity is also observed in tokamak experiments, e.g., in the experiments where the shear layer resides outside the last closed magnetic surface. ${ }^{31}$ These flows have only one shear layer, in contrast to the flows with even parity that possess a double shear layer. For the single layer flows, the natural boundary condition at $x=0, v(0)=0$, will not be consistent $(\Theta[v(0) \rightarrow 0] \rightarrow 0)$ with the poloidal momentum generation mechanism proposed in this Letter.

\section{Acknowledgments}

One of the authors (Y.Z. Zhang) acknowledges Prof. H.L. Berk for showing him his, as yet unpublished, manuscript (dated Jan. 1st, 1990) of the turbulence generated radial current in cold ion limit and of its possible role for the L-H transition. 


\section{References}

1. ASDEX TEAM, Nucl. Fusion 29, 1959 (1989).

2. R.J. Taylor, M.L. Brown, B.D. Fried, H. Grote, J.R. Liberati, G.J. Morales, and P. Pribyl, Phys. Rev. Lett. 63, 2365 (1989).

3. R.J. Groebner, K.H. Burrel, and R.P. Seraydarian, Phys. Rev. Lett. 64, 3015 (1990).

4. R.D. Stambaugh, S.M. Wolfe, R.D. Hawryluk, J.H. Harris, H. Biglari, S.C. Prager, R. J. Goldston, R.J. Fonck, T. Ohkawa, B.G. Logan, and E. Oktay, Phys. Fluids B 2, 2941 (1990).

5. K.H. Burrel, T.N. Carlstron, E.J. Doyle, P. Gohil, R.J. Groebner, T. Lehecka, N.C. Luhmann, H. Matsumoto, T.H. Osborne, W.A. Peebles, and R. Philipona, Phys. Fluids B 2, 1405 (1990).

6. Ch.P. Ritz, H. Lin, T.L. Rhodes, and A. Wootton, Phys. Rev. Lett. 65, 2543 (1990).

7. E.J. Doyle, R.J. Groebner, K.H. Burrel, P. Gohil, T. Lehecka, N.C. Luhmann, H. Matsumoto, T.H. Osborne, W.A. Peebles, and R. Philipona, Phys. Fluids B 3, 2300 (1991).

8. R. Philipona, E.J. Doyle, N.C. Luhmann, W.A. Peebles, C.L. Rettig, R.J. Groebner, K.H. Burrel, P. Gohil, H. Matsumoto, and R. Stambaugh, in Proceedings of the 18th European Conference on Controlled Fusion and Plasma Physics 1991, edited by P. Bachmann and D.C. Robinson (EPS Petti-Lancy, Switherland, 1991), Vol. 15C, Part I, pp. I-281 and A71.

9. K.R. Shaing, G.S. Lee, B.A. Carreras, W.A. Houlberg, and E.C. Crume, Jr., in Proceeding of the Tuelfth International Conference on Plasma Physics and Controlled Vuclear 
Fusion Research, 1988 (International Atomic Energy Agency, Vienna, 1989), Vol. II, p. 13 .

10. H. Biglari, P.H. Diamond, and P.W. Terry, Phys. Fluids B 2, 1 (1990).

11. K.R. Shaing, E.C. Crume, Jr., and W.A. Houlberg, Phys. Fluids B 2, 1492 (1990).

12. K.R. Shaing, Phys. Fluids B4, 290 (1992).

13. Y.Z. Zhang and S.M. Mahajan, Phys. Fluids B 4, 1385 (1992).

14. Y.Z. Zhang and S.M. Mahajan, submitted to Phys. Fluids.

15. S.-I. Itoh, and K. Itoh, Phys. Rev. Lett. 60, 2276 (1988).

16. A.B. Hassam, T.M. Antonsen, Jr., J.F. Drake, and C.S. Liu, Phys. Rev. Lett. 66, 309 (1991).

17. P.H. Diamond and Y.-B. Kim, Phys. Fluids B 3, 1626 (1991).

18. F. Hinton, Phys. Fluids B 3, 696 (1991).

19. J.F. Drake, J.M. Finn, P. Guzdar, V. Shapiro, V. Shevchenko, F. Waelbroeck, A.B. Hassam, C.S. Liu, and R. Sagdeev, Phys. Fluids B 4, 488 (1992).

20. H.L. Berk and K. Molvig, Phys. Fluids 26, 1385 (1983).

21. H.L.Berk, Private Communication.

22. J.F. Drake and A.B. Hassam, Phys. Fluids 24, 1262 (1981).

23. A.B. Hassam and J.F. Drake, Phys. Fluids 26, 133 (1983).

24. Y.Z. Zhang and S.M. Mahajan, Phys. Fluids B 4, 207 (1992). 
25. T.S. Hahm, P.H. Diamond, P.W. Terry, L. Garcia, and B.A. Carreras, Phys. Fluids 30, $1452(1987)$.

26. J.L. Johnson and J.M. Green, Plasma Physics 9, 611 (1967).

27. F.L. Hinton and J.A. Robertson, Phys. Fluids 27, 1243 (1984).

28. A simple analysis for the electrostatic detrapping can be found in H.L. Berk and A.A. Galeev, Phys. Fluids 10, 411 (1967). However, the real situation could be more complicated as the scale length of the shear flow is comparable with the ion banana width, and will be discussed elsewhere.

29. K.H. Burrell and the L to H Transition Physics Task Group, General Atomics, Bull. Am. Phys. Soc. 36 8T4, 2474 (1991).

30. R.J. Groebner, K.H. Burrell, P. Gohil, R.P. Seraydarian, J. Kim, T.C. Carlstron, R.R. Dominguez, G.M. Staebler, E.J. Doyle, R. Philipona, and C. Rettig, Bull. Am. Phys. Soc. $368 \mathrm{~T} 1,2474$ (1991).

31. H. Lin, R. Bengston, G.X. Li, A. Wootton, submitted to Phys. Rev. Lett. 


\section{Figure Captions}

1. A plot of the model nonlinear $\Theta[v]$ as a function of the poloidal velocity $v$ for the $\mathrm{L}-\mathrm{H}$ transition described by the differential system, Eq. (5).

2. Qualitative radial structure of the concave (a) and the convex (b) configuration for the same $\alpha\left(<\Theta\left[v^{*}\right]\right)$, where $v_{ \pm}$are the two solutions of $\alpha=\Theta\left[v_{ \pm}\right]$(cf. Fig. 1). 


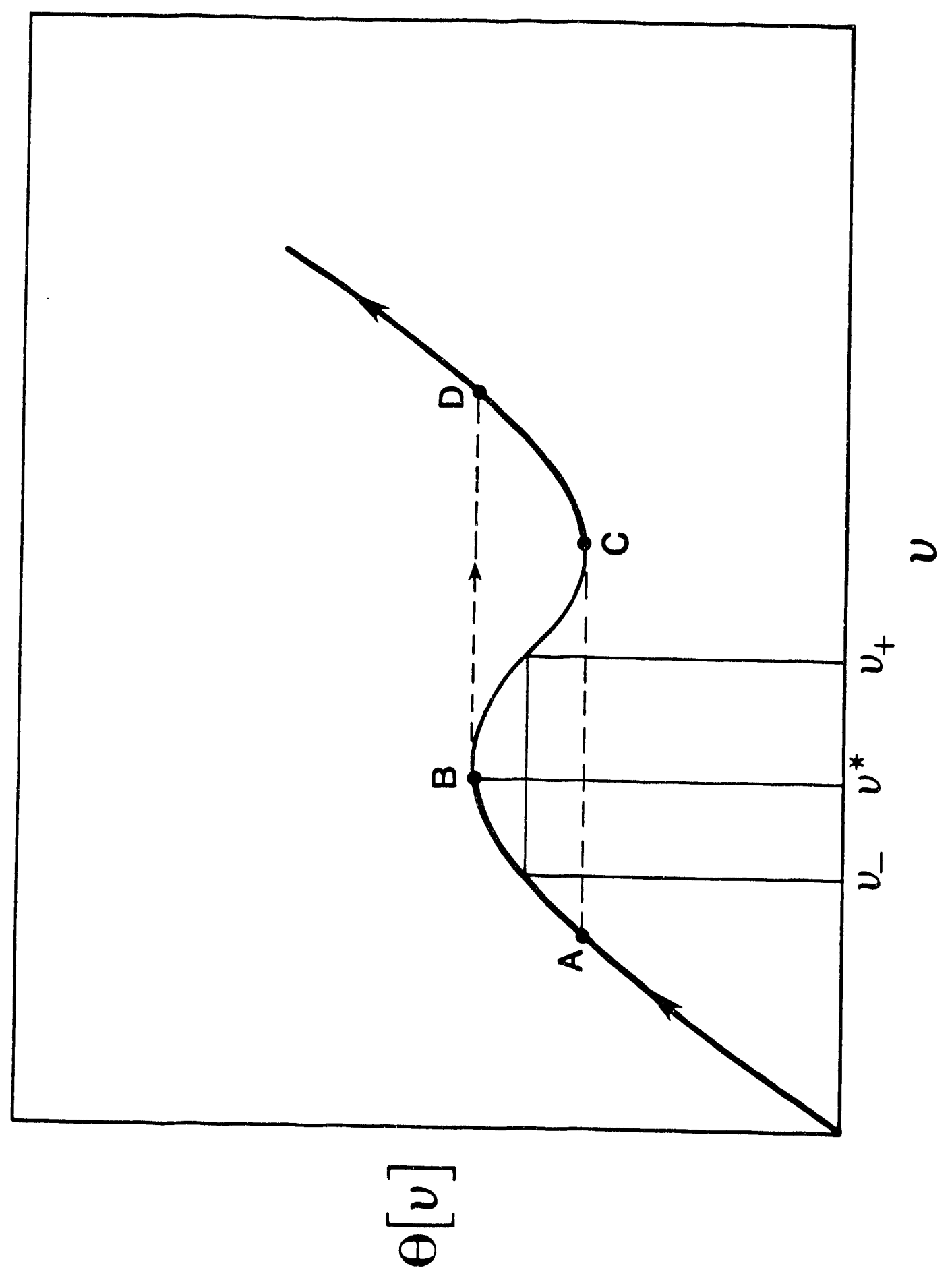

Fig. 1 


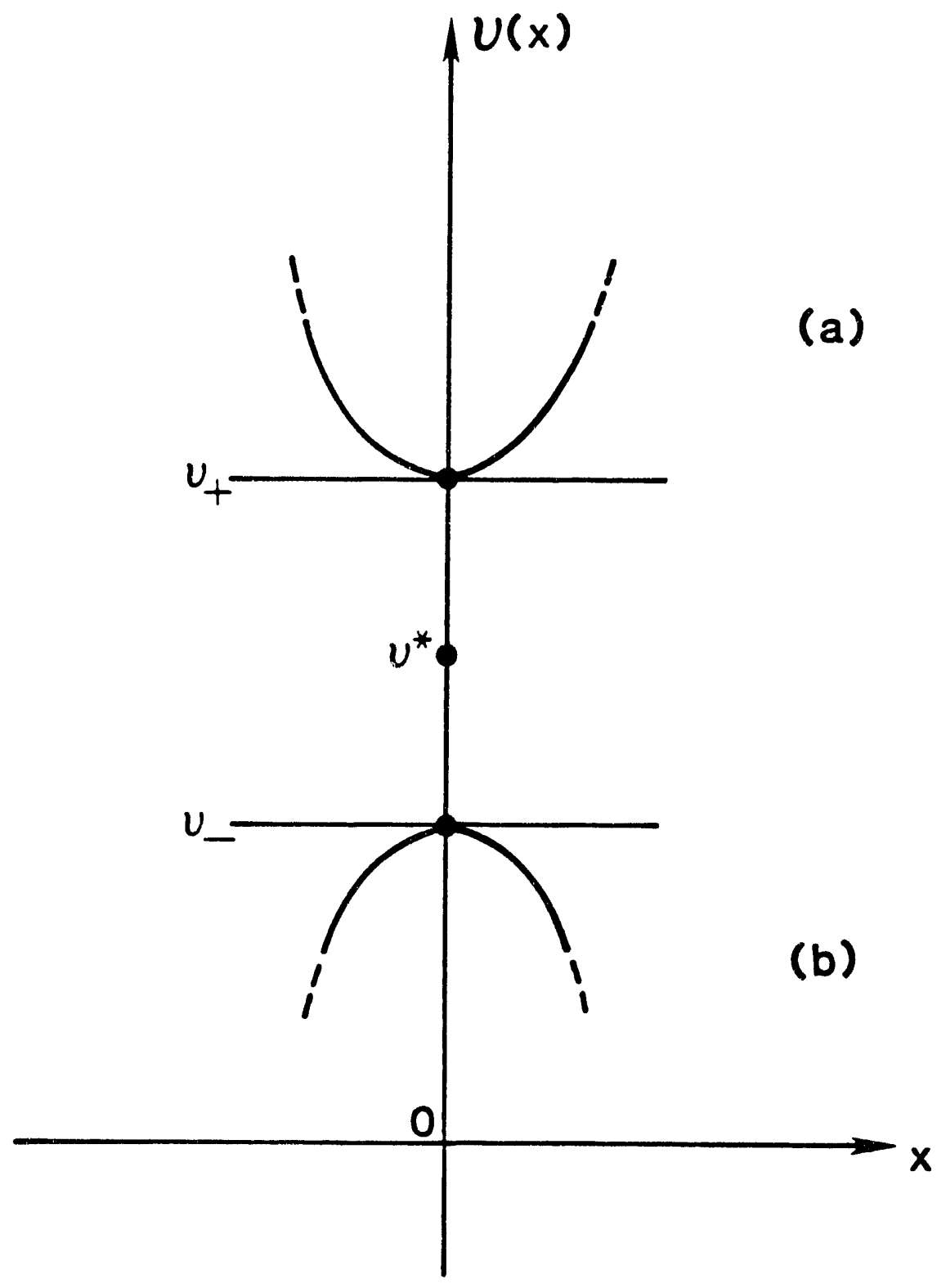

Fig. 2 

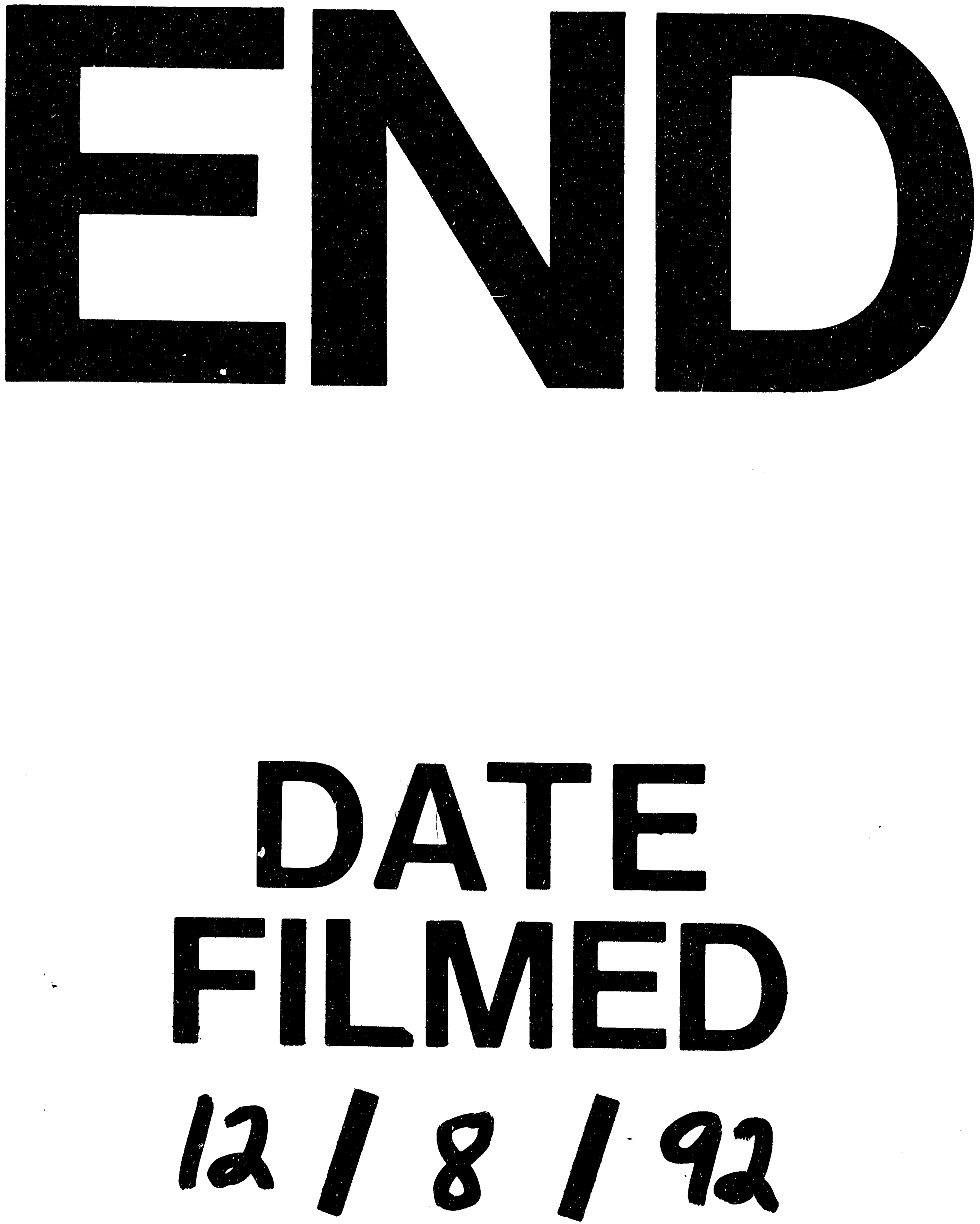
